\title{
Acciones para el desarrollo sustentable, estudiantes universitarios, su calidad de vida y un ambiente seguro
}

\section{Actions for sustainable development, university students, its quality of life and a safe environment}

MORÁN-DELGADO, Gabriela ${ }^{1}$, RANGEL-MONJARAS, Ma. Francisca², GARCÍA-GARCÍA, Jesús Alberto $^{1}$ y GARCÍA-CONTRERAS, Laura Patricia ${ }^{1}$

${ }^{I}$ Universidad Autónoma de Coahuila

${ }^{2}$ Universidad Lasalle Saltillo

ID ${ }^{\text {er }}$ Autor: Gabriela, Morán-Delgado / ORC ID: 0000-0001-8433-4216, Researcher ID Thomson: X-2070-2018, CVU CONACYT ID: 306238

ID $1^{\mathrm{er}}$ Coautor: Ma. Francisca, Rangel-Monjaras / ORC ID 0000-0003-1369-311X, Researcher ID Thomson: D-8211-2019

ID $2^{\text {do }}$ Coautor: Jesús Alberto, García-García

ID $3^{\text {er }}$ Coautor: Laura Patricia, García-Contreras

DOI: $10.35429 /$ JPDL.2019.15.5.15.21

Recibido 10 de Marzo, 2019; Aceptado 30 Junio, 2019

\section{Resumen:}

El objetivo es identificar las variables predicotras que contribuyen a la mejora de la calidad de vida y un ambiente seguro a través de acciones para promover el Desarrollo Sustentable. El estudio es no experimental con enfoque cuantitativo. La población 86,076 estudiantes de universidades públicas y privadas del Estado de Coahuila, una muestra de 305 casos para el presente estudio. El instrumento un cuestionario conformado por 291 ítems de los cuales se consideraron 40, 39 variables independientes que refieren a acciones que contribuyen al Desarrollo sustentable y una variable dependiente relacionada con la calidad de vida y el ambiente seguro. Se realizó un análisis de regresión múltiple el cual muestra que los estudiantes universitarios consideran acciones como el cuidado de la naturaleza, la equidad social, el ahorro de agua y fortalecer la identidad de las comunidades son predictoras de un ambiente seguro para la Calidad de vida. La contribución del estudio versa sobre la perspectiva de los estudiantes universitarios y las acciones propuestas para la mejora de su calidad de vida, un ambiente seguro y el Desarrollo sustentable.

Desarrollo Sustentable, Calidad de vida, Ambiente seguro

\begin{abstract}
:
The objective is to identify the predictive variables that contribute to the improvement of the quality of life and a safe environment through actions to promote Sustainable Development. The study is non-experimental with a quantitative approach. The population 86,076 students of public and private universities of the State of Coahuila, a sample of 305 cases for the present study. The instrument was a questionnaire made up of 291 items, of which 40, 39 independent variables were considered that refer to actions that contribute to sustainable development and a dependent variable related to the quality of life and the safe environment. A multiple regression analysis was carried out, which shows that university students consider actions such as caring for nature, social equity, saving water and strengthening the identity of communities that are predictors of a safe environment for quality of life. The contribution of the study is about the perspective of university students and the proposed actions to improve their quality of life, a safe environment and sustainable Development.
\end{abstract}

Sustainable Development, Quality of life, Safe environment

Citación: MORÁN-DELGADO, Gabriela, RANGEL-MONJARAS, Ma. Francisca, GARCÍA-GARCÍA, Jesús Alberto y GARCÍA-CONTRERAS, Laura Patricia. Acciones para el desarrollo sustentable, estudiantes universitarios, su calidad de vida y un ambiente seguro. Revista de Filosofía y Cotidianidad. 2019, 5-15: 15-21

\footnotetext{
* Correspondencia del Autor (gabriela.moran.delgado@uadec.edu.mx)

$\dagger$ Investigador contribuyendo como primer autor.
} 


\section{Introducción}

El movimiento de los Pueblos para la Educación de los Derechos Humanos en su página web declara que "los derechos humanos relacionados al medio ambiente se encuentran establecidos en los tratados básicos de derechos humanos e incluyen: el derecho a un medio ambiente seguro y saludable" (PDHRE, s.f.).

"En México se han reconocido los derechos de la naturaleza, lo que viene a revolucionar el derecho en nuestro país, y se suma al nuevo paradigma que surgió en el sur de nuestro continente" (Garza Grimaldo, 2015).

Según la Organización Mundial de la Salud (OMS), la calidad de vida es la percepción que un individuo tiene de su lugar en la existencia, en el contexto de la cultura y del sistema de valores en los que vive y en relación con sus objetivos, sus expectativas, sus normas, sus inquietudes.

Para comprender el concepto de ambiente seguro es necesario remitirse al Desarrollo Sustentable. Es el desarrollo que satisface las necesidades de las generaciones presentes sin comprometer la capacidad de las generaciones futuras para satisfacer sus propias necesidades, concepto que utilizó por primera vez en 1992 en la declaración de Rio sobre Medio ambiente y Desarrollo.

En lo que refiere a Equidad social, una publicación sobre Equidad e inclusión social de la Organización de Estados Americanos (2016) señala que los avances en materia de derechos civiles y políticos no se han correspondido con avances en materia de derechos económicos, sociales y culturales. Menciona también que la comunidad internacional reconoce que la equidad es un principio fundamental del desarrollo.

En un aporte desde la OEA se muestra que el continente americano no es el más pobre, pero sin duda si es el más desigual. En el mismo documento cita la definición de desigualdad en la cual existe una "dispersión de una distribución del ingreso, consumo, o en algún otro indicador relacionado con el bienestar" elaborada por el Banco Mundial (2011) (Muñoz-Pogossian B., 2016).
La OMS lleva tiempo señalando las importantes repercusiones de la integridad del medio ambiente en la salud y el Desarrollo de las personas. Declaran que en la actualidad existe mayor conocimiento, pruebas y comprensión sobre los modos y mecanismos que el clima y el cambio climático repercuten en la salud. Así también señala que en el caso particular de las ciudades en donde viven casi 4000 millones de personas, cerca de la mitad de la población mundial; esas personas se encentran expuestas a una serie de amenazas ambientales que resultan de las deficientes condiciones de la vivienda, el transporte, abastecimiento de agua potable, saneamiento y gestión de desechos. "La transición a un medio ambiente más sano y seguro exigirá una labor coordinada y la aportación de toda una panoplia de interlocutores tanto del sector salud como ajenos a él. Esta labor debe venir respaldada por datos convincentes y científicamente comprobados" (Neira María, 2017).

\section{Planteamiento}

La interrogante de esta investigación versa sobre ¿Cuáles son las acciones que los estudiantes de nivel superior consideran para contribuir al Desarrollo Sustentable y tener un ambiente seguro?

El objetivo es identificar las variables predicotras que contribuyen a la mejora de la calidad de vida y un ambiente seguro a través de acciones para promover el Desarrollo Sustentable. Las acciones que se promueven en este mundo globalizado han llevado a deteriorar con mayor rapidez el medio ambiente. Las políticas actuales han creado mayores brechas entre los grupos sociales, enfatizando las desigualdades sociales. El desperdicio y uso desmedido de los recursos naturales es una cuestión cultural hoy en día. Los gobiernos y algunos organismos internacionales llevan a cabo estrategias para moderar el deterioro del entorno natural, sin embargo es importante hacer conciencia en la sociedad para que se establezcan compromisos y lleven a cabo acciones en bien de la naturaleza y el entorno en que se vive. Toca parte importante de este compromiso a los jóvenes universitarios de realizar propuestas y llevar a cabo acciones que contribuyan a mejorar el ambiente y promover la cohesión social hacia medidas estratégicas que se concreticen en una mejor calidad de vida para todos los seres humanos. 


\section{Marco teórico}

Cada mujer, hombre, joven y niño tienen derecho a un medio ambiente seguro y saludable, como a otros derechos humanos fundamentales relacionados a un medio ambiente saludable: un alto estándar de salud, desarrollo ecológicamente sustentable, acceso a alimentación y agua potable, protección para la no discriminación, derecho a la educación y a compartir los beneficios del progreso (PDHRE, s.f.).

En relación a la identidad social, para Peris y Agut (2007) la identidad social se forma por la pertenencia a un grupo; que sea positiva o negativa dependerá de la valoración que el individuo hace de su grupo comparándolo con otros (Peris Pichastor R., 2007).

El cuidado de la naturaleza es una acción que requiere atención de todos los sectores de la sociedad"...a pesar de ser una problemática con un reconocimiento social creciente, estamos lejos de un consenso capaz de responder a los retos y riesgos ecológicos y sociales que impone el modelo cultural de dominación de la naturaleza que caracteriza la relación contemporánea entre las sociedades modernas y el mundo natural" (Garavito González, 2009).

Como parte esencial del cuidado de la naturaleza se encuentra la problemática del agua"...el tema del agua potable es un tema candente y siempre preocupante, que no solo afecta a los países de Tercer Mundo sino que también puede afectar a países desarrollados...tarifas de agua en bloque bien diseñadas son aquellas donde los bajos consumos pagan menos y obtienen subsidios en cuotas de conexiones.

Comprar agua a los distribuidores de agua embotellada siempre será más costoso que pagar por la suministrada a través del servicio municipal. Otras alternativas serán más onerosas en términos de los costos sociales y de oportunidad, en particular por el agua potable de calidad o el tener que recorrer largas distancias para la toma pública de agua, como ocurre en muchos países poco desarrollados" (Saldivar V., 2013). Para López y Barajas (2013) "la lógica de la globalización implica una menor participación del Estado dentro de la vida económica del país, por lo que en México no hay mucho que esperar al respecto.
Es la sociedad quien debe plantear sus propias propuestas de desarrollo y, desde lo local, comenzar a dinamizar a la economía para alcanzar sus propias expectativas de vida, de acuerdo a su idiosincrasia. Se necesita mayor acción colectiva, y para que esta exista deben estar presentes cohesividad, solidaridad, confianza, cooperación y objetivos en común (López Santiago N., 2013).

En un estudio que trata sobre la inclusión se concluye que inclusión y exclusión son procesos históricos y políticos mutuamente relacionados. Señala la presencia de un doble gesto que se encuentra implícito en la búsqueda de la "inclusión" y que pasan desapercibidas. Mientras que las narrativas teóricas hablan de igualar a los desiguales, o de incluir a los excluidos, las practicas para su inclusión se ubican en un trasfondo que las hace simultáneamente de desigualdad o exclusión (Aguilar Nery, 2013).

Dentro de las acciones para lograr una mayor equidad social, Muñoz (2014) propone que "Un transporte público urbano suficientemente accesible constituye uno de los principales condicionantes para la implantación de peajes urbanos, lo que permite garantizar, además de un adecuado nivel de equidad social en la movilidad, un elevado grado de aceptación social” (Muñoz Miguel, 2014).

En relación al cuidado del consumo y ahorro del agua Escobar y Ortega señalan que son varias las acciones emprendidas. "Se ha incluido el saneamiento y el acceso al agua potable como uno de los objetivos del milenio a través de cuyo mejoramiento se busca impactar de forma directa y positiva en los niveles de salud humana Naciones Unidas (2010), expectativa de vida y productividad; se han emprendido campañas de educación ambiental enfocadas a fomentar diversas opciones de ahorro de agua, mejorar su uso y almacenamiento, así como la forma de minimizar, desde los hogares, su contaminación...aún falta mucho como lo demuestra el que no se hayan alcanzado las metas que en materia de cobertura de agua y saneamiento se plantearon a nivel mundial para 2015, según conclusiones del seguimiento realizado por la OMS en (2014) (Escobar Neira C., 2014). 
"La nueva ley de protección de la Tierra mantiene el derecho a un medio ambiente sano (antropocentrismo), pero además, reconoce los derechos de la naturaleza (biocentrismo); reivindica el valor primordial de la vida: los seres vivos tienen el mismo derecho a existir, a desarrollarse y a expresarse con autonomía, merecen respeto al tener el mismo valor...De seguir con ese exacerbado consumismo voraz, necesitamos tres Tierras para continuar con ese ritmo. En los últimos 50 años, se consumió lo equivalente a lo consumido en la historia de la humanidad" (Garza Grimaldo, 2015).

En un estudio realizado en España que refiere a la presencia de desigualdades en el acceso a prestaciones sanitarias Urbanos (2016), señala que las propuestas para lograr la equidad afectan a distintos ejes del sistema de salud. El estudio concluye que la equidad en el acceso a las prestaciones sanitarias debe definirse en sentido amplio, para incluir desde la igualdad del derecho a la protección de la salud hasta la igualdad en la obtención de una atención sanitaria efectiva y de calidad (Urbanos Garrido, 2016).

Los indicadores de progreso para la medición de los derechos contemplados en el protocolo Adicional a la Convención Americana sobre Derechos Humanos, indicadores de un instrumento único que contribuye a los esfuerzos estatales para traducir compromisos y obligaciones en materia de derechos, en particular a seguridad social, salud, educación, trabajo y derechos sindicales, alimentación adecuada, medio ambiente sano, y a los beneficios de la cultura, en políticas públicas que faciliten el goce de esos derechos (MuñozPogossian B., 2016).

Otro aspecto relevante para lograr una equidad social es el campo de la salud. A este tema Haeberer (2019) afirma que "La mortalidad cardiovascular está inversamente asociada con el nivel educativo. Esta desigualdad afecta más a la mortalidad prematura por causas cardiacas, especialmente entre mujeres. Su monitorización podría orientar la Estrategia de Salud Cardiovascular del sistema Nacional de Salud, para reducir la desigualdad en la primera causa de muerte" (Haeberer, 2019).
Los estudios científicos que demuestran los beneficios para la salud del contacto regular con espacios Naturales y la realización de actividad física en ellos son cada vez más precisos y concluyentes, e incorporan numerosos aspectos relacionados con la salud física y mental (Castell, 2019).

\section{Metodología}

El estudio es un diseño no experimental con un enfoque cuantitativo, ya que las variables se midieron en forma numérica. Se realizó una revisión de diversos documentos para lograr la conceptualización de las variables. El instrumento está conformado de 241 variables, de las cuales solamente se consideraron 49 para el estudio; 48 de ellas se muestran como variables independientes ya que son las que refieren a las acciones que los estudiantes consideran que se deben llevar a efecto para contribuir al desarrollo sustentable, y una variable dependiente que corresponde al eje de desarrollo humano y calidad de vida.

El instrumento se validó con expertos así como con la obtención del alpha de cronbach para su mayor nivel de confiabilidad, de lo que se obtuvo un resultado de (.87) y un alpha estandarizado de (.90). La población está conformada por 86,076 estudiantes de nivel superior en el Estado de Coahuila, según datos registrados en la Secretaría de Educación Pública, y la muestra que se empleó para el estudio fueron 305 sujetos ambos sexos y que estudian una carrera en instituciones de Educación Superior en la ciudad de Saltillo, capital del Estado de Coahuila. Se consideraron 9 centros educativos de nivel Superior, y los instrumentos fueron aplicados con un muestreo no probabilístico accidental (Castañeda Jiménez, 2011), pues solamente respondieron los estudiantes que aceptaron por voluntad propia. Los tratamientos estadísticos que se realizaron fueron un análisis de frecuencias para caracterizar a la población y un análisis de regresión múltiple, lo que proporciono los resultados que se consideraron para elaborar las conclusiones del presente estudio.

\section{Resultados}

Los resultados del análisis de frecuencias muestran que la población en lo que se refiere al género presenta un equilibrio de $50 \%$ hombres y $50 \%$ mujeres. 


\begin{tabular}{|l|r|r|r|r|}
\hline Categorías Sujetos & $\begin{array}{l}\text { Acumulado de } \\
\text { sujetos }\end{array}$ & Porcentaje & $\begin{array}{l}\text { Porcentaje } \\
\text { acumulado }\end{array}$ \\
\hline Femenino & 152 & 152 & 50 & 50 \\
\hline Masculino & 152 & 304 & 50 & 100 \\
\hline
\end{tabular}

Tabla 1 Análisis de frecuencias del género al que pertenecen los encuestados

Fuente: Elaboración Propia

Así también en el mismo análisis de frecuencias se muestran los porcentajes de edad de los sujetos encuestados mismos que indican que la mayor parte se concentran en los 20,21, 19,22 y 18 años en ese orden de mayor a menor concentración.

\begin{tabular}{|l|r|r|r|r|}
\hline Categorías & \multicolumn{1}{c}{ Sujetos } & \multicolumn{1}{c|}{$\begin{array}{c}\text { Acumulado } \\
\text { de sujetos }\end{array}$} & Porcentaje & $\begin{array}{c}\text { Porcentaje } \\
\text { acumulado }\end{array}$ \\
\hline 17 & 7 & 7 & 2.3 & 2.3 \\
\hline 18 & 33 & 40 & 10.82 & 13.11 \\
\hline 19 & 52 & 92 & 17.05 & 30.16 \\
\hline 20 & 74 & 166 & 24.26 & 54.43 \\
\hline 21 & 58 & 224 & 19.02 & 73.44 \\
\hline 22 & 35 & 259 & 11.48 & 84.92 \\
\hline 23 & 21 & 280 & 6.89 & 91.8 \\
\hline 24 & 10 & 290 & 3.28 & 95.08 \\
\hline 25 & 9 & 299 & 2.95 & 98.03 \\
\hline 26 & 4 & 303 & 1.31 & 99.34 \\
\hline 27 & 1 & 304 & 0.33 & 99.67 \\
\hline 36 & 1 & 305 & 0.33 & 100 \\
\hline
\end{tabular}

Tabla 2 Análisis de frecuencias de la edad a la que pertenecen los encuestados

Fuente: Elaboración Propia

En las Instituciones de Educación Superior que participaron en la encuesta, el análisis de frecuencias muestra que en su mayor parte los encuestados se concentran en estudiantes que cursan sus estudios en UA de C, ITS, , UTC y ULSA, en ese orden de mayor a menor concentración.

\begin{tabular}{|c|c|c|c|c|}
\hline Categorías & Sujetos & $\begin{array}{l}\text { Acumulado } \\
\text { de sujetos }\end{array}$ & Porcentaje & $\begin{array}{l}\text { Porcentaje } \\
\text { acumulado }\end{array}$ \\
\hline UVM & 36 & 36 & 11.8 & 11.8 \\
\hline ULSA & 31 & 67 & 10.16 & 21.97 \\
\hline ITS & 49 & 116 & 16.07 & 38.03 \\
\hline UVIZ & 12 & 128 & 3.93 & 41.96 \\
\hline ENEF & 29 & 157 & 9.51 & 51.47 \\
\hline UAdeC & 81 & 238 & 26.56 & 78.03 \\
\hline UTC & 35 & 273 & 11.48 & 89.51 \\
\hline ENRE & 10 & 283 & 3.28 & 92.79 \\
\hline UPRA & 22 & 305 & 7.21 & 100 \\
\hline
\end{tabular}

Tabla 3 Análisis de frecuencias del centro educativo al que pertenecen los encuestados

Fuente: Elaboración Propia
Universidad del Valle de México (UVM), Universidad Lasalle (ULSA), Instituto Tecnológico de Saltillo (ITS), Universidad Vizcaya de las Américas (UVIZ), Escuela Normal de Educación Física (ENEF), Universidad Autónoma de Coahuila (UA de C), Universidad Tecnológica de Coahuila (UTC), Escuela Normal Regional de Educación Superior (ENRE), Universidad Politécnica de Ramos Arizpe (UPRA).

En los resultados del análisis de regresión múltiple se encontró que las variables predictoras que los estudiantes de nivel superior consideran para tener un ambiente seguro son $\mathrm{el}$ ahorro del agua, la equidad social, la identidad de las comunidades y el cuidado de la naturaleza, con un R2 de (0.418).

Por lo que se infiere que los estudiantes encuestados consideran que acciones como el cuidar el consumo de agua, la equidad social, mantener la identidad en las comunidades y el cuidado de la Naturaleza son acciones que favorecen para tener un ambiente seguro.

\begin{tabular}{|l|l|l|l|l|l|l|}
\hline \multicolumn{7}{|c|}{ Variable independiente: Ambiente Seguro } \\
\hline R2=0.4187 & $\begin{array}{l}\text { Variables } \\
\text { nte de } \\
\text { regresió } \\
\text { n b(i) }\end{array}$ & $\begin{array}{l}\text { Coeficie } \\
\text { Stánd } \\
\text { art } \\
\text { Sb(i) }\end{array}$ & $\begin{array}{l}\text { Valor de } \\
\text { Prueba } \\
\text { T } \\
\text { HO:B(i) } \\
==\end{array}$ & $\begin{array}{l}\text { Nivel de } \\
\text { probabili } \\
\text { dad }\end{array}$ & $\begin{array}{l}\text { Recha H0 } \\
\text { za } \\
\text { al } \\
5 \% ?\end{array}$ & $\begin{array}{l}\text { Pode } \\
\text { r de } \\
\text { Prue } \\
\text { ba al } \\
5 \%\end{array}$ \\
\hline $\begin{array}{l}\text { Ahorro } \\
\text { del agua }\end{array}$ & 0.143 & $\begin{array}{l}0.059 \\
8\end{array}$ & 2.392 & 0.0175 & $\mathrm{Si}$ & $\begin{array}{l}0.66 \\
39\end{array}$ \\
\hline $\begin{array}{l}\text { Equidad } \\
\text { social }\end{array}$ & 0.1561 & 3 & 2.632 & 0.009 & $\mathrm{Si}$ & $\begin{array}{l}0.74 \\
62\end{array}$ \\
\hline $\begin{array}{l}\text { Identidad } \\
\text { de las } \\
\text { comunida } \\
\text { des }\end{array}$ & -0.1766 & 2 & -2.628 & 0.0091 & $\mathrm{Si}$ & $\begin{array}{l}0.74 \\
48\end{array}$ \\
\hline $\begin{array}{l}\text { Cuidado } \\
\text { de 1a } \\
\text { naturaleza }\end{array}$ & 0.2142 & $\begin{array}{l}0.071 \\
4\end{array}$ & 3.001 & 0.003 & $\mathrm{Si}$ & $\begin{array}{l}0.84 \\
85\end{array}$ \\
\hline
\end{tabular}

Tabla 4 Análisis de regresión múltiple considerando la variable Ambiente Seguro como dependiente

Fuente: Elaboración Propia

\section{Conclusiones}

Un ambiente seguro incluye beneficios que son considerados en la propia declaración de derechos humanos; la educación, la salud, el trabajo, la igualdad social, la alimentación, el desarrollo ecológicamente sustentable y los beneficios del progreso son algunos de los derechos que benefician a los seres humanos. 
El cuidado de la naturaleza es una acción que corresponde a todos. La protección a la tierra reconocido ya como un derecho en la "Ley de Protección de la Tierra" señala el valor primordial de la vida y de todos los seres vivos; sin embargo, se resaltan las acciones de cosumismo exacervado de los recursos naturales, como lo refiere Garza (2015); así también Garavito (2009) señala que estamos lejos de responder a los retos y riesgos ecológicos y sociales impuestos por el modelo cultural de dominación de la Naturaleza.

Los resultados del estudio muestran como los jóvenes estudiantes de nivel superior consideran relevante el cuidado de la naturaleza como una acción que contribuye al desarrollo sustentable y que además, como señalan estudios científicos repercute en beneficios para la salud física y mental (Castell 2019).

El agua es otro de los elementos indispensables para el ambiente seguro. Se han implementado algunas estrategias para el cuidado del consumo, algunas de ellas realizadas por organismos internacionales como la ONU; campañas de educación ambiental enfocadas al ahorro, uso y almacenamiento del líquido, sin embargo no se han alcanzado los objetivos planteados, tal y como lo señala Escobar (2014). Este problema no solo afecta a países del Tercer mundo, sino también a países con un cierto nivel de desarrollo (Saldivar 2013).

López (2013) señala que la lógica de la globalización implica una menor participación del estado y alude a la organización de la sociedad para plantear sus propias propuestas de desarrollo social; alcanzar sus propias expectativas de vida de acuerdoa su idiosincracia. La identidad social es relevante para alcanzar esta cohesión social, dicha identidad, para Peris (2007) depende de la valoración que el individuo hace de su grupo social. Sin embargo la Convención Americana sobre Derechos Humanos, en sus indicadores refiere a los esfuerzos estatales para traducir compromisos y obligaciones en materia de derechos: aspectos como la seguridad social, salud, educación, alimentación y medio ambiente.
Estudios como el de Aguilar (2013) concluyen que la inclusión y exclusión son procesos históricos y políticos mutuamente relacionados y que mientras en teoría se habla de igualar a los desiguales o incluir a los excluidos, en la práctica para la inclusión se hace un trasfondo de desigualdad o exclusión. Otros autores como Muñoz (2014) declara que acciones como la implementación de un transporte urbano suficuentemente accesible permite alcanzar un nivel adecuado de equidad social; así también Garrido (2016) concluye que la equidad en el acceso a las prestaciones sanitarias debe definirse en sentido amplio para incluir desde la igualdad de derecho a la protección de la salud hasta la igualdad en la obtención de una atenión sanitaria efectiva y de calidad. Haeberer (2019), muestra en su estudio una desigualdad en la mortalidad cardiovascular asociada con el nivel educativo, especialmente en mujeres.

Se puede concluir que las acciones para un Desarrollo Sustentable y mejor calidad de vida propuestas por los estudiantes de nivel superior, resultado de este estudio estan estrecahamente relacionadas. El ahorro del agua es una medida indispensable en el cuidado de la Naturaleza; para que exista una mayor equidad social es importante la administración ordenada de los recursos naturales y materiales; y, es la identidad social la que nos invita a plantearnos objetivos y compromisos que contribuyan al bienestar de toda la humanidad y de la naturaleza. Es esta una propuesta de un ambiente seguro derivado de acciones para un Desarrollo sustentable y por tanto una mejor calidad de vida.

\section{Referencias}

Castañeda Jiménez, J. (2011). metodología de la Investigación. México, México: McGraw-Hill.

Castell, C. (2019). Naturaleza y salud: una alianza necesaria. Gaceta Sanitaria, https://doi.org/10.1016/j.gaceta.2019.05.016.

López Santiago N., B. G. (julio-diciembre de 2013). Identidad y desarrollo: El caso de la subregión alta Mixe de Oaxaca. Península, III(2), 9-37. 
Aguilar Nery, J. (24 de enero de 2013). programas educativos compensatorios en México. Problemas de equidad y conocimiento. Perfiles Educativos, XXXVII(147), 183-200.

Escobar Neira C., O. A. (enero de 2014). Efectividad de tres estrategias de educación ambiental en el ahorro del agua en una universidad. Revista Latinoamericana de Medicina Conductual, 4(1), 41-58.

Garavito González, L. (enero-diciembre de 2009). La indagación sociológica contemporánea sobre la "naturaleza". Territorios(20-21), 207-217.

Garza Grimaldo, J. G. (febrero de 2015). Los derechos de la naturaleza en México. Revista Mexicana de Ciencias Agrícolas, 1, 181-190.

Haeberer, M. e. (3 de septiembre de 2019). Desigualdades sociales en la mortalidad cardiovascular en España desde una perspectiva interseccional. Revista Española de Cardiología, 72(9), 701-794.

Muñoz Miguel, J. (7 de Noviembre de 2014). Disponibilidad y accesibilidad en el transporte público madrileño como garantía de equidad social ante la aplicación de un peaje urbano para la ciudad de Madrid. Investigaciones Europeas de Dirección y Economía de la Empresa.(20), 47-54.

Muñoz-Pogossian B., B. A. (2016). Equidad e inclusión social: Superando desigualdades hacia sociedades más inclusivas. Washington DC, USA: OEA.

Neira María, P. M.-L.-U. (20 de octubre de 2017). Rumbo a un medio ambiente más sano. Recuperado el septiembre de 2019, de Organización Mundial de la salud: https://www.who.int/es/news-

room/commentaries/detail/towards-a-healthierand-safer-environment

PDHRE. (s.f.). PDHRE. Recuperado el septiembre de 2019, de https://www.pdhre.org/rights/environmentsp.html

Peris Pichastor R., A. N. (diciembre de 2007). Evolución conceptual de la Identidad social. El retorno de los procesos emocionales. Revista electrónica de motivación y Emoción, X(26-27).
Saldivar V., A. (julio-agosto de 2013). Gobernanza multidimensional del agua: la Directiva del Agua Europea. Dificultades de su aplicación. Economía informa(381), 74-90.

Urbanos Garrido, R. (20 de marzo de 2016). La desigualdad en el acceso a las prestaciones sanitarias. Propuestas para lograr la equidad. Gaceta Sanitaria, 25-30. 